\title{
Enhancement of the Sensitivity of Gas Sensor Based on Microstructure Optical Fiber
}

\author{
Monir MORSHED ${ }^{1 *}$, Md. Imran HASAN ${ }^{2}$, and S. M. Abdur RAZZAK ${ }^{3}$ \\ ${ }^{1}$ Department of Information and Communication Technology, Mawlana Bhashani Science and Technology University, \\ Tangail-1902, Bangladesh \\ ${ }^{2}$ Department of Electronics \& Telecommunication Engineering, Rajshahi University of Engineering \& Technology, \\ Rajshahi-6204, Bangladesh \\ ${ }^{3}$ Department of Electrical \& Electronic Engineering, Rajshahi University of Engineering \& Technology, Rajshahi-6204, \\ Bangladesh \\ *Corresponding author: Monir MORSHED ～E-mail: monirmorshed.mbstu@gmail.com
}

\begin{abstract}
This paper proposes the design and characterization of microstructure optical fiber for gas sensing applications. The aim is to detect toxic and colorless gases over a wide transmission band covering $0.80 \mu \mathrm{m}$ to $2.00 \mu \mathrm{m}$ wavelength. Numerical investigation is carried out by using the finite element method (FEM). The numerical study shows that sensitivity of the proposed sensor is moderately increased by introducing four non-circular holes around the defected core of photonic crystal fiber and the confinement loss is also reduced. Furthermore, we confirm that increasing the diameter of central air core and size of the non-circular holes can improve the relative sensitivity and the confinement loss is reduced by increasing the diameter of air holes in the cladding. The enhancement of the relative sensitivity is more than $27.58 \%(0.1323$ to 0.1688$)$ at the wavelength $\lambda=1.33 \mu \mathrm{m}$ that is the absorption line of methane $\left(\mathrm{CH}_{4}\right)$ and hydrogen fluoride (HF) gases. The confinement loss of the fiber is $1.765 \times 10^{-8} \mathrm{~dB} / \mathrm{m}$.
\end{abstract}

Keywords: Confinement loss, gas sensor, hollow core photonic crystal fiber, microstructure fiber, relative sensitivity

Citation: Monir MORSHED, Md. Imran HASAN, and S. M. Abdur RAZZAK, "Enhancement of the Sensitivity of Gas Sensor Based on Microstructure Optical Fiber,"Photonic Sensors, 2015, 5(4): 312-320.

\section{Introduction}

Photonic crystal fibers (PCFs) consist of periodically arranged microscopic cylindrical air holes that run along the entire length of fiber [1]. The PCF attracts the researchers due to its unique features and noble optical characteristics such as high birefringence, high nonlinearity, the endlessly single mode, flexible chromatic dispersion and the wider design space, and also its vast area applications of communication and sensing devices [1-8]. The design flexibility of PCF fascinates us to design PCF based gas sensors for chemical and biological sensing compared with conventional fibers [9-11].

Index guiding (IG) PCFs [12-15] and photonic band gap (PBG) PCFs $[3,10,16]$ are used to implement gas sensors. A gas sensor based on hollow core PBGPCFs is proposed in $[12,14-17]$ to confine more light into the core which contains gas

Received: 2 March 2015 / Revised: 9 August 2015

(C) The Author(s) 2015. This article is published with open access at Springerlink.com

DOI: $10.1007 / \mathrm{s} 13320-015-0247-y$

Article type: Regular 
samples. Since in this fiber, fraction of total power is enhanced by the power of hollow core, the sensitivity is significant [12, 14-17]. However, it has two fundamental limitations: narrow transmission spectral band and lack of precise control of waveguide parameters for maintaining the band gap effect [14-17]. To overcome these limitations, index guided PCF is considered as a potential candidate. But the relative sensitivity of this fiber is very low due to poor interaction between light penetrating into the holes in the cladding and the gas samples inside the holes $[14,15]$.

Many research works have reported different structures of PCFs to increase the sensing capability of index guided fibers [12-17]. Park et al. [14] introduced high index $\mathrm{GeO}_{2}$ doped $\mathrm{SiO}_{2}$ glass ring around the central air hole to improve the relative sensitivity and to reduce the confinement loss. In this paper, fraction of evanescent field is enhanced by increasing the diameter of central air hole, hence the sensitivity is improved and kept the diameter smaller than cladding holes diameters to physical realization of the effective index guiding. An optimize structure of [14] was proposed in [18] to get the better sensitivity and lower confinement loss. Also, it introduced a new structure with hexagonal holes in the inner ring instead of circular holes to significantly increase the sensitivity. This paper shows the relative sensitivity diagram for both of the hexagonal and circular holes but the confinement loss curve is shown for circular holes only. According to the results of above papers, it has been observed that both the relative sensitivity and confinement loss are improved but not significant for a gas sensor. To increase the relative sensitivity and reduce the confinement loss, much more work has to be done. An effective way to increase the performance of a gas sensor is to design a new structure.

In 2013, an evanescent wave based PCF with four non-circular large air holes in the cladding was proposed by Zhang et al. [19] for gas sensing application. It showed that non-circular holes nearest core region helps to increase the relative sensitivity and reduce the confinement loss due to very high air filling fraction of about $93 \%$.

In this paper, it proposes an optimum structure of prior PCF [18] by introducing four non-circular air holes instead of six hexagonal holes around a hollow high index $\mathrm{GeO}_{2}$-doped silica ring to increase the relative sensitivity and to decrease the confinement loss.

\section{Geometries of the proposed PCF}

Figure 1 shows the transverse cross section of the proposed structure which contains five air-hole rings in the cladding. The diameter of holes in two outermost rings, diameter of holes in two innermost rings, width of the non-circular holes, width of the high index GeO2-doped silica ring, hole to hole space (pitch), and diameter of central air core are $d_{2}$, $d_{1}, W, W_{d}, \Lambda$, and $d_{c}$, respectively. A defected hollow high index $\mathrm{GeO}_{2}$-doped silica ring is considered at the center of fiber around an air core whose width is varies according to the diameter of air core.

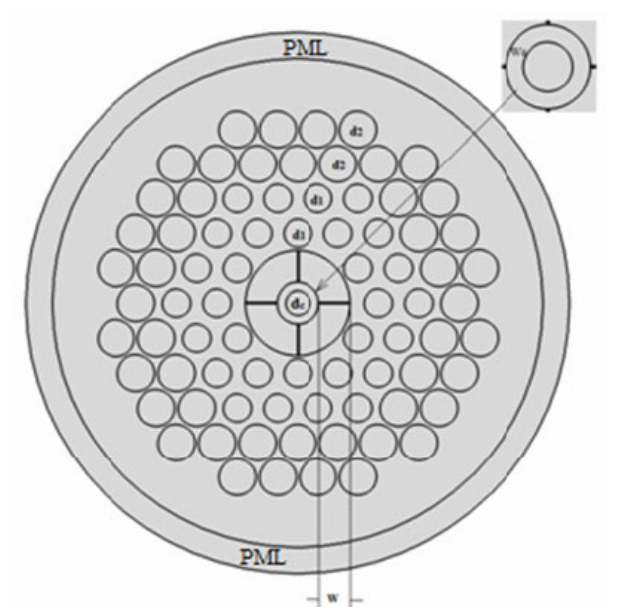

Fig.1 Transverse cross section of the designed PCF shows pitch $\Lambda$, central hole diameter $d_{c}$, width of non-circular holes $W$, width of the doped ring $W_{d}$, diameter of holes in rings 2 and $3 d_{1}$, diameter of holes in rings 4 and $5 d_{2}$.

In [18], it was shown that the outer cladding had greater impact on confinement loss and sensitivity is greatly depended on the diameter of holes in the inner ring closer to the core region. We observe in our investigation that the diameter of holes in cladding and width of non-circular holes has strong 
impact on confinement loss. Hence, $d_{1}, d_{2}$, and $W$ are considered larger to reduce confinement loss. Also, larger $W$ helps to increase the interaction between evanescent waves and gases which are located inside the holes. Hence, the relative sensitivity is increased.

\section{Numerical analysis and equations}

To simulate the optical properties of the proposed PCFs, a finite element method (FEM) is used for solving Maxwell's equation due to its proven reliability which provides good approximation of the circular PCF structure [14, 20-22].

Due to the finite number of air holes in the cladding, light can leak among the air holes which are called the leakage loss or confinement loss. On the other hand, it is the ability to confine the light into core region. However, the confinement loss can be omitted for infinite number of air holes in the cladding. But it is not practically feasible. Hence, to compute the leakage loss, an effective boundary condition is needed, which produces no reflection at the boundary. Perfectly, matched layers (PMLs) are the most efficient absorption boundary condition for this purpose $[23,24]$. We consider the circular PML [14], where electric field satisfies the Maxwell's equations:

$$
\nabla \times\left(\mu_{r}^{-1} \nabla \times \mathbf{E}\right)-k_{0}^{2}\left(\varepsilon_{r}-\mathrm{j} \frac{\sigma}{\omega \varepsilon_{0}}\right) \cdot \mathbf{E}=0
$$

where $\mu_{r}$ and $\varepsilon_{r}$ are relative permeability and relative permittivity, respectively. $k_{0}$ is the free-space wave number, $\omega$ is the angular frequency, and $\sigma$ is the electric conductivity. The free space wave number is given $k_{0}=n_{\text {eff }} \beta$, here $\beta$ is the propagation constant and $n_{\text {eff }}$ is an effective refractive index of mode. Variation of effective index according to the wavelength is called material dispersion, which can be calculated by using Sellmeier equations which gives an accurate value of index for the material silica, $\mathrm{SiO}_{2}$, and germane-silicate glass, $\mathrm{GeO}_{2}-\mathrm{SiO}_{2}$ [25].

The confinement loss $(\mathrm{dB} / \mathrm{m})$ is determined from the imaginary part of effective mode index as follows [18, 23]

$$
L_{c}=8.686 k_{0} \operatorname{Im}\left[n_{\text {eff }}\right]
$$

where $\operatorname{Im}\left[n_{\text {eff }}\right]$ is the imaginary part of the effective mode index.

Beer-Lambert law $[18,26]$ is used to evaluate the absorbance of evanescent field by the gas samples. The relationship between gas concentration and optical intensity is given by the following equation

$$
I(\lambda)=I_{0}(\lambda) \exp \left[-r \alpha_{m} l c\right]
$$

where $I$ and $I_{0}$ are the output light intensities with and without the presence of gas being detected, respectively, $\alpha_{m}$ is the absorption coefficient of the gas being measured and is a function of wavelength, $l$ is the length of the PCF used for gas detection (interaction length), $c$ is the gas concentration, and $r$ is a relative sensitivity coefficient defined as $[18$, 26]

$$
r=\frac{n_{s}}{\operatorname{Re}\left[n_{\text {eff }}\right]} f
$$

where $n_{s}$ are refractive index of gas species considered almost equal to $1, \operatorname{Re}\left[n_{\text {eff }}\right]$ is the real part of the effective mode index, and $f$ is the fraction of the total power located in the holes that can be expressed as

$$
f=\frac{\int_{\text {holes }}\left(E_{x} H_{y}-E_{y} H_{x}\right) d x d y}{\int_{\text {total }}\left(E_{x} H_{y}-E_{y} H_{x}\right) d x d y}
$$

where $E_{x}, E_{y}, H_{x}$, and $H_{y}$ are the transverse electric and magnetic fields of the guided mode. These transverse fields, $E x, E_{y}, H_{x}$, and $H_{y}$ and effective index of mode $n_{\text {eff }}$ can be calculated by solving Maxwell's equation by using the FEM method.

For remote detection, it is desirable to use low loss optical fiber in the near infrared region having minimum transmission losses $(<1 \mathrm{~dB} / \mathrm{km})$. Our main focus is on the methane $\left(\mathrm{CH}_{4}\right)$ and hydrogen fluoride (HF) gases having the absorption line at the wavelength of $1.33 \mu \mathrm{m}$. We have tried to improve the relative sensitivity and confinement loss at this wavelength. Furthermore, for the other wavelength our proposed PCF has better results compared with 
prior PCFs. The indices difference between the core and cladding can be greater by increasing the doping percent of $\mathrm{GeO}_{2}$, and it assists to reduce the confinement losses. As like as prior PCF, we have used $\mathrm{GeO}_{2}-\mathrm{SiO}_{2}$ with $\mathrm{GeO}_{2}$ concentration of $19.3 \%$ mol that makes an index difference of $1.89 \%$ between pure silica and GeO2-doped silica [18].

\section{Numerical results and discussions}

Figure 2 shows the fundamental mode field distribution at the operating wavelength of $1.33 \mu \mathrm{m}$ for our proposed PCFs. From this figure, it is seen that the Gaussian distribution of fields are uniform all through the $\mathrm{GeO}_{2}$-doped silica ring and fundamental mode are tightly bounded in that ring. Also, it shows that evanescent field strongly interacts with the samples of holes near the core region that makes the sensitivity very high.

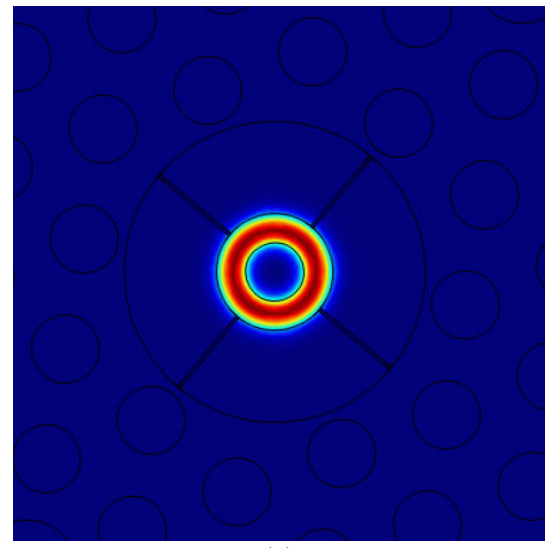

(a)



(b)

Fig. 2 Modal intensity distribution of proposed PCF at the wavelength of $1.33 \mu \mathrm{m}$ for the geometrical parameters $d_{c}=1.4 \mu \mathrm{m}, \Lambda=2.3 \mu \mathrm{m}, d_{1}=1.4 \mu \mathrm{m}$, and $d_{2}=2 \mu \mathrm{m}$ : (a) $2 \mathrm{D}$ view and (b) $3 \mathrm{D}$ view.
The relative sensitivity and confinement loss curves of the optimum design of the proposed PCF for non-circular holes, hexagonal holes, and prior PCF with hexagonal holes [18] are shown in Figs. 3 and 4 . The optimized parameters for the proposed PCF is $\Lambda=2.3 \mu \mathrm{m}, W=1.8 \mu \mathrm{m}, d_{c}=1.4 \mu \mathrm{m}, W_{d}=0.5 \mu \mathrm{m}$, $d_{1} / \Lambda=0.782$, and $d_{2} / \Lambda=0.956$. It can be seen that the designed PCF has a relative sensitivity of $16.88 \%$ and the confinement loss of the fiber is $1.765 \times 10^{-8} \mathrm{~dB} / \mathrm{m}$. Also, it is clear that the proposed PCF has a higher relative sensitivity and lower confinement loss compared with prior PCFs.

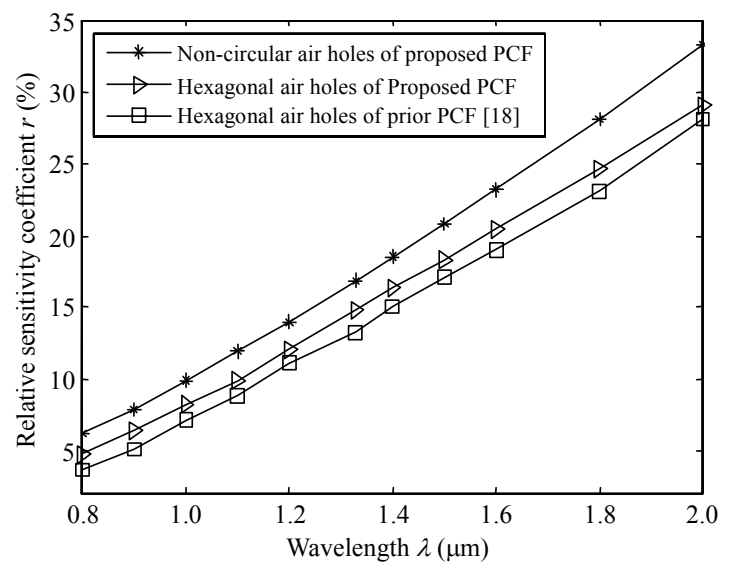

Fig. 3 Relative sensitivity versus wavelength for the proposed PCF (non-circular and hexagonal) for the optimum design parameters: $W=1.8 \mu \mathrm{m}, d_{c}=1.4 \mu \mathrm{m}, W_{d}=0.5 \mu \mathrm{m}$, $d_{1} / \Lambda=0.782$, and $d_{2} / \Lambda=0.956$ and prior PCF (hexagonal).

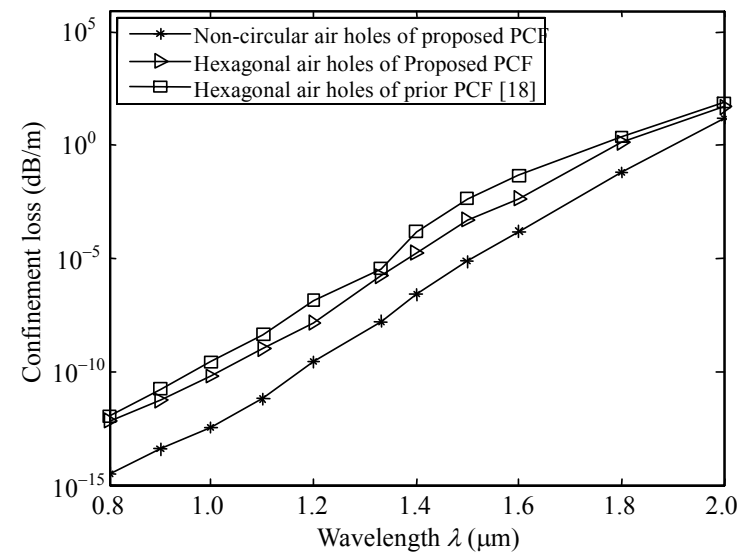

Fig. 4 Confinement loss versus wavelength for the proposed PCF (non-circular and hexagonal) for the optimum design parameters: $W=1.8 \mu \mathrm{m}, d_{c}=1.4 \mu \mathrm{m}, W_{d}=0.5 \mu \mathrm{m}, d_{1} / \Lambda=0.782$, and $d_{2} / \Lambda=0.956$ and prior PCF (hexagonal).

To overcome the critical trade-off between the relative sensitivity and confinement loss, the 
parameters of the proposed PCF are optimized. For the optimization of the parameters, a simple technique is applied. Firstly, the thickness of PML is fixed to $10 \%$ of the radius of the proposed fiber by the PML test for efficient calculation of confinement loss but it has no significant effect on the relative sensitivity. The thickness of circular PML is $1.5 \mu \mathrm{m}$. Then, $W$ is varied by $0.8 \mu \mathrm{m}, 1.2 \mu \mathrm{m}, 1.6 \mu \mathrm{m}$, and $1.8 \mu \mathrm{m}$ while other parameters are kept constant to $\Lambda=2.3 \mu \mathrm{m}, d_{c}=1.2 \mu \mathrm{m}, W_{d}=0.6 \mu \mathrm{m}, d_{1} / \Lambda=0.61$, and $d_{2} / \Lambda=0.87$ which are shown in Figs.5 and 6. The relative sensitivity at the wavelength of $1.33 \mu \mathrm{m}$ is $10.4 \%, 11.7 \%, 11.75 \%$, and $11.758 \%$, respectively; the confinement loss is $3.5 \times 10^{-2} \mathrm{~dB} / \mathrm{m}$, $3.15 \times 10^{-3} \mathrm{~dB} / \mathrm{m}, \quad 1.2 \times 10^{-4} \mathrm{~dB} / \mathrm{m}$, and $9.08 \times 10^{-6} \mathrm{~dB} / \mathrm{m}$, respectively.

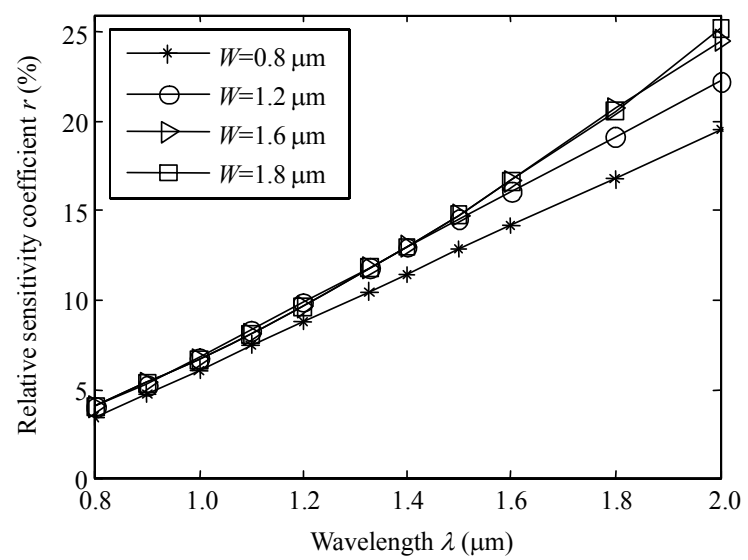

Fig. 5 Relative sensitivity coefficient of the proposed PCF shows the impact of $W$ when other geometrical parameters are kept constant.

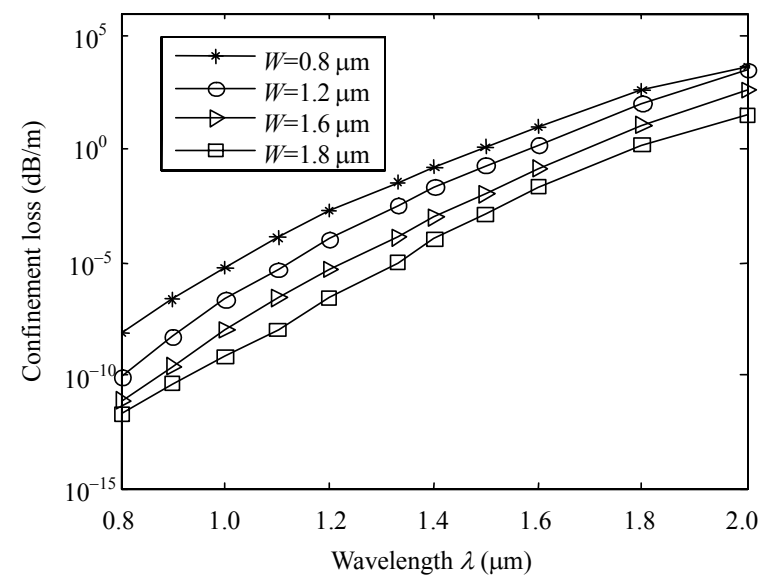

Fig. 6 Confinement loss of the proposed PCF shows the impact of $W$ when other geometrical parameters are kept constant.
The response of the real part of the effective refractive index over wavelength is shown in Fig. 7. From the figure, it is clear that the effective refractive index decrease with an increase in $d_{c}$ and lowering $W_{d}$. So the indices difference between the core and cladding is reduced. As a result, more evanescent field is penetrated into the non-circular air holes around the core region, which helps to increase the fraction of power of holes that lead to higher relative sensitivity. In this case, central air core $d_{c}$ is changed by $1.2 \mu \mathrm{m}, 1.3 \mu \mathrm{m}, 1.4 \mu \mathrm{m}$, and $1.5 \mu \mathrm{m}$ when other geometrical parameters $\Lambda=2.3 \mu \mathrm{m}, W=1.8 \mu \mathrm{m}, d_{1} / \Lambda=0.61$, and $d_{2} / \Lambda=0.87$ are kept constant.



Fig. 7 Index versus wavelength of the proposed PCF.

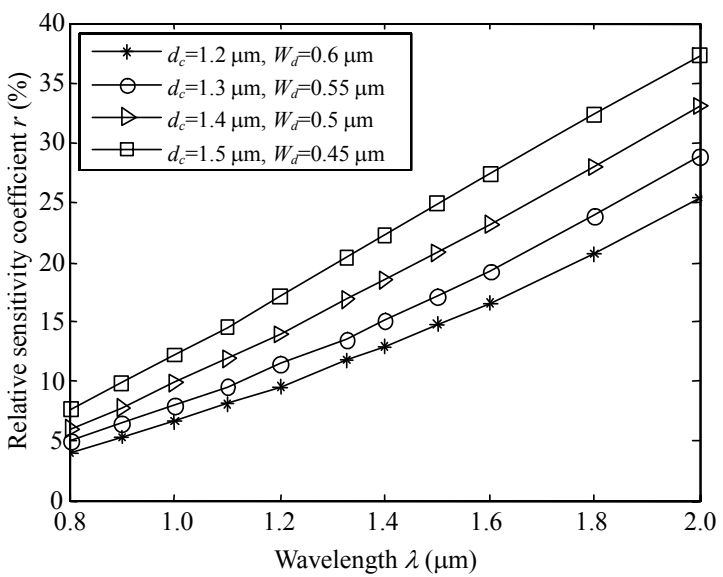

Fig. 8 Relative sensitivity coefficient of the proposed PCF shows the impact of $d_{c}$ and $W_{d}$ when other geometrical parameters are kept constant.

Figures 8 and 9 show the impacts of $d_{c}$ and $W_{d}$ on the relative sensitivity and the confinement loss 
properties of our structures when other parameters are fixedly setting to $\Lambda=2.3 \mu \mathrm{m}, W=1.8 \mu \mathrm{m}$, $d_{1} / \Lambda=0.61$, and $d_{2} / \Lambda=0.87$. Now $d_{c}$ is chosen as $1.2 \mu \mathrm{m}, 1.3 \mu \mathrm{m}, 1.4 \mu \mathrm{m}$, and $1.5 \mu \mathrm{m}$ and corresponding $W_{d}$ is set as $0.6 \mu \mathrm{m}, 0.55 \mu \mathrm{m}, 0.5 \mu \mathrm{m}$, and $0.45 \mu \mathrm{m}$. At $\lambda=.33 \mu \mathrm{m}$, the calculated relative sensitivity is $11.758 \%, 13.5 \%, 16.88 \%$, and $20.43 \%$, respectively; the confinement loss of the fiber is $9.08 \times 10^{-6} \mathrm{~dB} / \mathrm{m}, 1.80 \times 10^{-5} \mathrm{~dB} / \mathrm{m}, 3.1 \times 10^{-3} \mathrm{~dB} / \mathrm{m}$, and $1.922 \times 10^{-1} \mathrm{~dB} / \mathrm{m}$, respectively. From the figures, it is observed that both the relative sensitivity and confinement loss are increased with the air core diameter. But to maintain the trade-off between the two properties, the value of $d_{c}$ is adjusted to $1.4 \mu \mathrm{m}$.

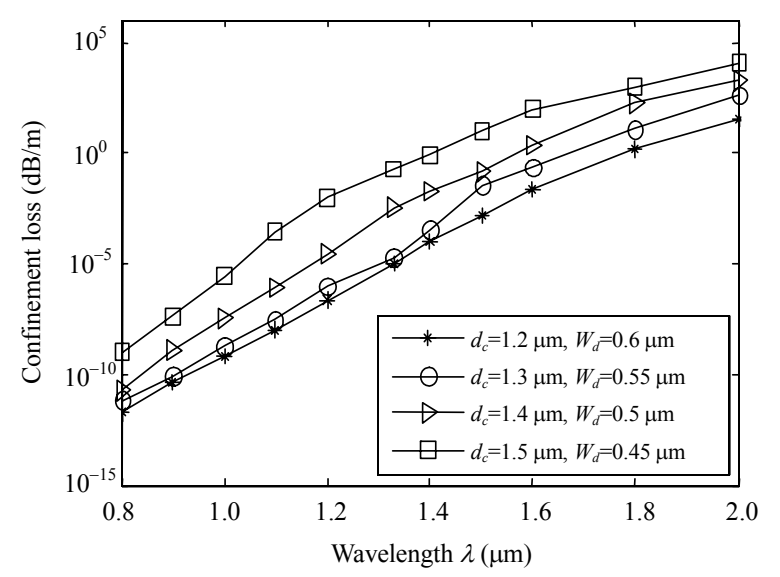

Fig. 9 Confinement loss of proposed PCF shows the impact of $d_{c}$ and $W_{d}$ when other geometrical parameters are kept constant.



Fig. 10 Relative sensitivity coefficient of proposed PCF shows the impact of $d_{1} / \Lambda$ when other geometrical parameters are kept constant.

Figures 10 and 11 reveal the impacts of $d_{1} / \Lambda$ on the relative sensitivity and confinement loss while other parameters are kept constant to $\Lambda=2.3 \mu \mathrm{m}$, $W=1.8 \mu \mathrm{m}, d_{c}=1.4 \mu \mathrm{m}, W_{d}=0.5 \mu \mathrm{m}$, and $d_{2} / \Lambda=0.956$. Now $d_{1} / \Lambda$ is chosen as $0.61,0.652,0.70$, and 0.783 , respectively. It is shown that increasing the value of $d_{1} / \Lambda$ corresponding relative sensitivity of the proposed PCF is increased about $16.8735 \%$, $16.874 \%, 16.875 \%$, and $16.876 \%$ at the wavelength of $1.33 \mu \mathrm{m}$ and the corresponding calculated confinement loss is $3.1 \times 10^{-3} \mathrm{~dB} / \mathrm{m}, 6.77 \times 10^{-4} \mathrm{~dB} / \mathrm{m}$, $1 \times 10^{-4} \mathrm{~dB} / \mathrm{m}$, and $1.52 \times 10^{-5} \mathrm{~dB} / \mathrm{m}$, respectively.



Fig. 11 Confinement loss of the proposed PCF shows the impact of $d_{1} / \Lambda$ when other geometrical parameters are kept constant.



Fig. 12 Relative sensitivity of the proposed PCF shows the impact of $d_{2} / \Lambda$ when other geometrical parameters are kept constant.

Figures 12 and 13 exhibit the effects of $d_{2} / \Lambda$ on the relative sensitivity and confinement loss. Now $d_{2} / \Lambda$ is varied by $0.87,0.91$, and 0.956 while other 
parameters $\Lambda=2.3 \mu \mathrm{m}, \quad W=1.8 \mu \mathrm{m}, \quad d_{c}=1.4 \mu \mathrm{m}$, $W_{d}=0.5 \mu \mathrm{m}, d_{1} / \Lambda=0.783$ are kept constant. The calculated relative sensitivity at $\lambda=1.33 \mu \mathrm{m}$ is $16.88 \%$ for all values of $d_{2} / \Lambda$; the confinement loss is $1.52 \times 10^{-5} \mathrm{~dB} / \mathrm{m}, 1.48 \times 10^{-7} \mathrm{~dB} / \mathrm{m}$, and $1.765 \times 10^{-8} \mathrm{~dB} / \mathrm{m}$, respectively.

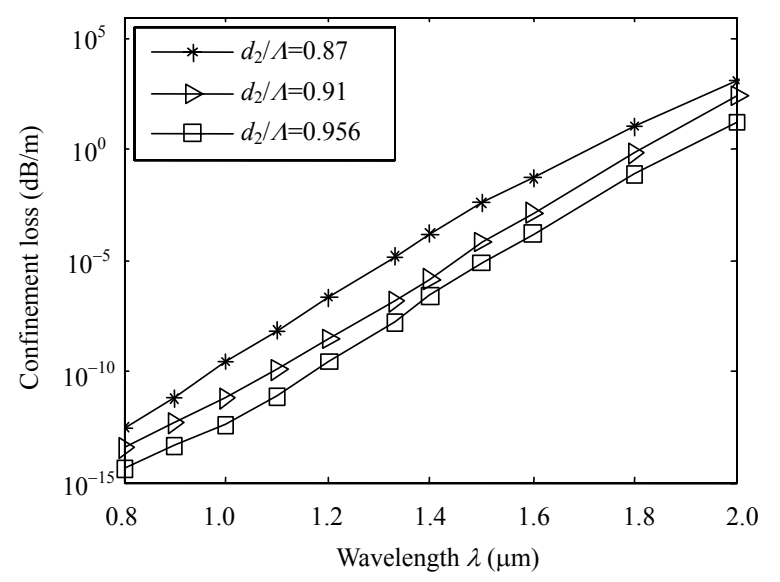

Fig. 13 Confinement loss of the proposed PCF shows the impact of $d_{2} / \Lambda$ when other geometrical parameters are kept constant.

From Figs. 10 and 12, it is clear that air holes in the cladding having the diameters $d_{1}$ and $d_{2}$ have not significant effect on the relative sensitivity, but the confinement loss is reduced with an increase in $d_{1} / \Lambda$ and $d_{2} / \Lambda$ shown in Figs. 11 and 13.

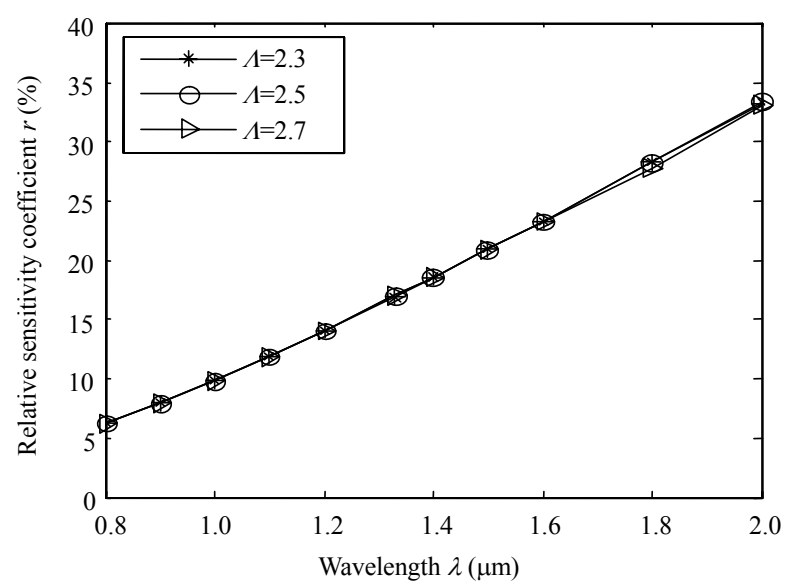

Fig. 14 Relative sensitivity coefficient of the proposed PCF shows the impact of $\Lambda$ when other geometrical parameters are kept constant.

Figures 14 and 15 present the effects of $\Lambda$ on the relative sensitivity and confinement loss. Now, $\Lambda$ is changed by $2.3 \mu \mathrm{m}, 2.5 \mu \mathrm{m}$, and $2.7 \mu \mathrm{m}$ when other parameters are kept constant to $W=1.8 \mu \mathrm{m}$, $d_{c}=1.4 \mu \mathrm{m}, W_{d}=0.5 \mu \mathrm{m}, d_{1}=1.8 \mu \mathrm{m}$, and $d_{2}=2.2 \mu \mathrm{m}$. The computed relative sensitivity at $\lambda=1.33 \mu \mathrm{m}$ is $16.88 \%$ for all values of $\Lambda$ and confinement loss is $1.765 \times 10^{-8} \mathrm{~dB} / \mathrm{m}, 1.8 \times 10^{-4} \mathrm{~dB} / \mathrm{m}$, and $1.2 \times 10^{-3} \mathrm{~dB} / \mathrm{m}$, respectively. We can observe from the figures that the relative sensitivity is not affected by the change in pitch but the confinement loss increases dramatically with an increase in the value of $\Lambda$.

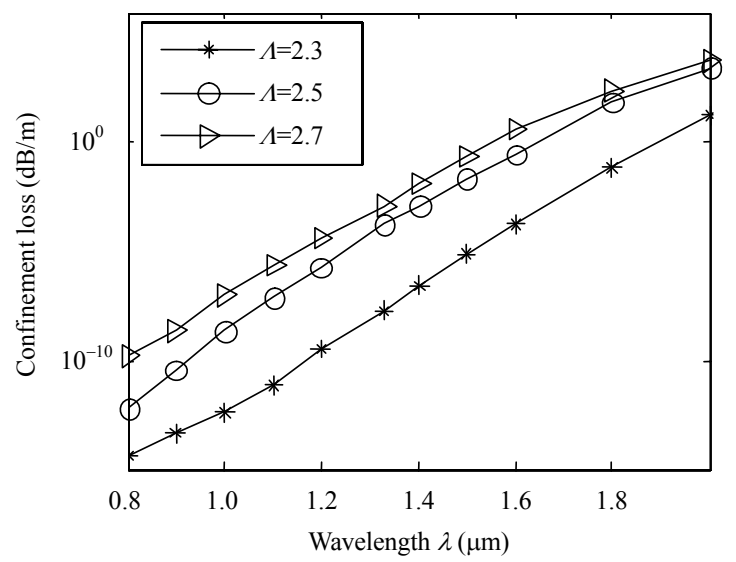

Fig. 15 Confinement loss of the proposed PCF shows the impact of $\Lambda$ when other geometrical parameters are kept constant.

According to the above discussion to act as a gas sensor, the optimized parameters of the proposed PCF is found as $W=1.8 \mu \mathrm{m}, d_{c}=1.4 \mu \mathrm{m}, W_{d}=0.5 \mu \mathrm{m}$, $d_{1} / \Lambda=0.783, d_{2} / \Lambda=0.956$, and $\Lambda=2.3 \mu \mathrm{m}$ at which the relative sensitivity coefficient is $16.88 \%$ and confinement loss is $1.765 \times 10^{-8} \mathrm{~dB} / \mathrm{m}$.

Table 1 compares the relative sensitivity coefficient and confinement loss properties of proposed structure and other reported fibers. It clearly indicates that the designed fiber could provide better performance and overcome the critical trade-off between the confinement loss and sensitivity compared with prior PCFs.

Table1 Comparison between the proposed PCF and prior PCFs for sensing application at $\lambda=1.33 \mu \mathrm{m}$.

\begin{tabular}{ccccc}
\hline PCFs & $r(\%)$ & $\begin{array}{c}L_{\mathrm{c}} \\
(\mathrm{dB} / \mathrm{m})\end{array}$ & $\begin{array}{c}\text { Air-hole shape } \\
\text { in the first ring }\end{array}$ & $\begin{array}{c}\text { Number of holes } \\
\text { in the first ring }\end{array}$ \\
\hline$[14]$ & 5.09 & 1.25 & Circle & 6 \\
{$[18]$} & 9.9 & $6.85 \times 10^{-5}$ & Circle & 6 \\
{$[18]$} & 13.3 & $3.77 \times 10^{-6}$ & Hexagonal & 6 \\
Proposed PCFs & 16.88 & $1.765 \times 10^{-8}$ & Non-circle & 4 \\
\hline
\end{tabular}

Finally, the proposed PCFs may not be easy to fabricate. However, this proposed structure can be 
fabricated by the present available technology due to technological advancement in the fabrication of PCFs. Several methods have been developed for the fabrication of microstructured fibers such as stack and draw [27], sol-gel casting [28], drilling [9], and extrusion [29] methods. Stack and draw methods are bounded to closest-packed geometries such as triangular or honeycomb lattices and cannot easily generate circular patterns. The drilling methods permit adjustment of both the hole size and spacing, but are generally limited to a small number of holes and restricted to circular shapes. Extrusion techniques provide design freedom, but are typically limited to soft glasses for which the material loss values are exceedingly high. Bise et al. [28] developed the sol-gel technique to fabricate the PCFs with any structure and provide the freedom to adjust the air-hole size, shape, and spacing. In addition, the sol-gel casting method provides design flexibility that will be essential for the proposed PCF.

\section{Conclusions}

The effective mode index, transverse electric, and magnetic fields of a new PCF structure with four non-circular air holes around a hollow high index $\mathrm{GeO}_{2}$-doped silica ring has been presented. The FEM with PML has been used as the numerical tool. It has been shown by numerical simulation that the proposed structure warrants a relative sensitivity, $r$, of $16.88 \%$ for an air core diameter $d_{c}=1.40 \mu \mathrm{m}$ which is significantly larger than those of other PCFs. It is expected that the proposed structure compared with other PCFs can provide easier fabrication due to less number of air holes nearest the core region. PCFs with these properties would be useful for practical applications in toxic and colorless gas detection.

Open Access This article is distributed under the terms of the Creative Commons Attribution License which permits any use, distribution, and reproduction in any medium, provided the original author(s) and source are credited.

\section{References}

[1] P. Russell, "Photonic crystal fibers," Science, 2003, 299(5605): 358-362.

[2] M. Nielsen, C. Jacobsen, N. Mortensen, J. Folkenberg, and H. Simonsen, "Low-loss photonic crystal fibers for transmission systems and their dispersion properties," Optics Express, 2004, 12(7): 1372-1376.

[3] B. Temelkuran, S. D. Hart, G. Benoit, J. D. Joannopoulos, and Y. Fink, "Wavelength-scalable hollow optical fibers with large photonic band gaps for CO2 laser transmission," Nature, 2002, 420(6916): 650-653.

[4] H. Du, "SERS-based photonic crystal fiber sensing platform," in Proc. SPIE, vol. 6083, pp. 74, 2006.

[5] P. Bing, J. Li, Y. Lu, Z. Di, and X. Yan, "Theoretical and experimental researches on a PCF-based SPR sensor," Optoelectronics Letters, 2012, 8(4): 0245-0248.

[6] Y. Yuan, Z. Guo, and L. Ding, "Influence of metal layer on the transmitted spectra of SPR-based optical fiber sensor," Optoelectronics Letters, 2010, 6(5): 346-349.

[7] S. Olyaee and A. A. Dehghani, "High resolution and wide dynamic range pressure sensor based on two-dimensional photonic crystal," Photonic Sensors, 2012, 2(1): 92-96.

[8] S. Olyaee and A. A. Dehghani, "Nano-pressure sensor using high quality photonic crystal cavity resonator," in 2012 8th International Symposium on Communication Systems, Networks \& Digital Signal Processing (CSNDSP), Poznan, pp. 1-4, 2012.

[9] M. N. Petrovich, A. Brakel, F. Poletti, K. Mukasa, E. Austin, V. Finazzi, et al., "Microstructured fibers for sensing applications," Proceedings of SPIE - The International Society for Optical Engineering, 2005, 6005(1): 60050E-1-60050E-15.

[10] T. Ritari, J. Tuominen, H. Ludvigsen, J. Petersen, T. Sørensen, T. Hansen, et al., "Gas sensing using air-guiding photonic bandgap fibers," Optics Express, 2004, 12(17): 4080-4087.

[11] J. M. Fini, "Microstructure fibers for optical sensing in gases and liquids," Measurement Science and Technology, 2004, 15(6): 1120-1128.

[12] X. Yu, Y. C. Kwok, N. A. Khairudin, and P. Shum, "Absorption detection of cobalt (II) ions in an index-guiding microstructured optical fiber," Sensors and Actuators: B Chemical, 2009, 137(2): 462-466.

[13] C. Martelli, J. Canning, D. Stocks, and M. J. Crossley, "Water-soluble porphyrin detection in a pure-silica photonic crystal fiber," Optics Letters, 2006, 31(14): 2100-2102.

[14] J. Park, S. Lee, S. Kim, and K. Oh, "Enhancement of chemical sensing capability in a photonic crystal fiber with a hollow high index ring defect at the 
center," Optics Express, 2011, 19(3): 1921-1929.

[15] S. Olyaee and A. Naraghi, "Design and optimization of index-guiding photonic crystal fiber gas sensor," Photonic Sensor, 2013, 3(2): 131-136.

[16] S. Smolka, M. Barth, and O. Benson, "Highly efficient fluorescence sensing with hollow core photonic crystal fibers," Optics Express, 2007, 15(20): 12783-12791.

[17] Y. L. Hoo, W. Jin, J. Ju, and H. L. Ho, "Numerical investigation of a depressed-index core photonic crystal fiber for gas sensing," Sensors and Actuators B: Chemical, 2009, 139(2): 460-465.

[18] S. Olyaee, A. Naraghi, and V. Ahmadi, "High sensitivity evanescent-field gas sensor based on modified photonic crystal fiber for gas condensate and air pollution monitoring," Optik - International Journal for Light and Electron Optics, 2014, 125(1): 596-600.

[19] L. Zhang, G. Ren, and J. Yao, "A new photonic crystal fiber gas sensor based on evanescent wave in terahertz wave band: design and simulation," Optoelectronics Letters, 2013, 9(6): 0438-0440.

[20] S. Olyaee and F. Taghipour," Doped-core octagonal photonic crystal fiber (O-PCF) with ultra-flattened nearly zero dispersion and low confinement loss in a wide wavelength range," Fiber and Integrated Optics, 2012, 31(3): 178-185.

[21] K. Saitoh and M. Koshiba, "Full-vectorial imaginary-distance beam propagation method based on a finite element scheme: application to photonic crystal fibers," IEEE Journal of Quantum
Electronics, 2002, 38(7): 927-933.

[22] F. Brechet, J. Marcou, D. Pagnoux, and P. Roy, "Complete analysis of the characteristics of propagation into photonic crystal fibers, by the finite element method," Optical Fiber Technology, 2000, 6(2): 181-191.

[23] Md. Selim Habib, Md. Samiul Habib, S. M. Abdur Razzak, and Md. Anwar Hossain, "Proposal for highly birefringent broadband dispersion compensating octagonal photonic crystal fiber," Optical Fiber Technology, 2013, 19(5): 461-467.

[24] K. Saitoh and M. Koshiba, "Leakage loss and group velocity dispersion in air-corephotonic bandgap fibers," Optics Express, 2003, 11(23): 3100-3109.

[25] T. Izawa and S. Sudo, Optical fibers: materials and fabrication. Berlin, Germany: Springer-Verlag, 1987.

[26] Y. L. Hoo, W. Jin, C. Shi, H. L. Ho, D. N. Wang, and S. C. Ruan, "Design and modeling of a photonic crystal fiber gas sensor," Applied Optics, 2003, 42(18): 3509-3515.

[27] J. Broeng, D. Mogilevstev, S. E. Barkou, and A. Bjarklev, "Photonic crystal fibers: a new class of optical waveguides," Optical Fiber Technology, 1999, 5(3): 305-330.

[28] R. T. Bise and D. J. Trevor, "Sol-gel derived microstructured fiber: fabrication and characterization," in Optical Fiber Communications Conference (OFC), Anaheim, U. S. A., 2005.

[29] H. Ebendorff-Heidepriem, P. Petropoulos, S. Asimakis, V. Finazzi, R. Moore, K. Frampton, et al., "Bismuth glass holey fibers with high nonlinearity," Optics Express, 2004, 12(21): 5082-5087. 